\title{
SOME DIFFERENTIAL EQUATIONS ON CLOSED MANIFOLDS
}

\author{
RODRIGO A. RESTREPO
}

Generalizations of the ordinary, linear, homogeneous differential equations with constant coefficients can be obtained by replacing the usual operation of differentiation by more general differential operators. Special cases of these generalizations are obtained by considering equations of the form

$$
\sum_{k=0}^{n} a_{k}(\delta d)^{k} \alpha=0, \quad a_{0} \neq 0,
$$

or

$$
\sum_{k=0}^{n} a_{k}(d \delta)^{k} \alpha=0, \quad a_{0} \neq 0,
$$

where $a_{0}, \cdots, a_{n}$ are constants, $d$ and $\delta$ are the differential operators used by de Rham and Kodaira [1], and $\alpha$ is a differential form defined on a manifold $M$. Assuming that $M$ is a closed, orientable Riemannian manifold of class $C^{\infty}$, it will be shown below that equations (1) and (2) have solutions only for some special values of $a_{0}, \cdots, a_{n}$, and their solutions are always linear combinations of the solutions of the eigenvalue problems

$$
\delta d \alpha-a \alpha=0, \quad a \neq 0
$$

and

$$
d \delta \alpha-a \alpha=0, \quad a \neq 0
$$

respectively. Thus there is an obvious analogy with the familiar case where the solutions are built up from the solutions of the eigenvalue problem $-y^{\prime \prime}-a y=0$. However, in the closed manifold the eigenvalues must be positive, and the repeated roots of the auxiliary equations, associated with (1) and (2) contribute no extra solutions for the differential equation.

These results will be established only for equation (1). Analogous methods can be applied to (2), or this equation can be reduced to (1) by means of the operator*. For the study of (1) it is convenient to introduce the concept of a field system, defined as follows:

Received by the editors February 8, 1960.

1 The relevant results from [2] are listed in the appendix to this paper. 
Definition. A field system $A$ is a doubly infinite sequence of differential forms $\alpha^{n}, n=0, \pm 1, \cdots$, that for each $n$ satisfy one of the following conditions:

(i) $\alpha^{n}$ is closed and $\alpha^{n+1}=\delta \alpha^{n}$,

(ii) $\alpha^{n}$ is co-closed and $\alpha^{n+1}=d \alpha^{n}$.

It is shown in [2] that any exact or co-exact form determines a unique field system in the manifolds under consideration. For example, on the unit circle, a field system is given by $\alpha^{2 n}=\sin x, \alpha^{2 n+1}$ $=\cos x d x$, all $n$. In other manifolds it may be possible to obtain only an infinite sequence with $n \geqq 0$. For example, in the four-dimensional space of special relativity, the electro-magnetic potential $\alpha$, field $\beta$ and current $\gamma$ form the first three elements of such a sequence since

$$
\begin{array}{ll}
\delta \alpha=0, & d \alpha=\beta, \\
d \beta=0, & \delta \beta=\gamma, \\
\delta \gamma=0 . &
\end{array}
$$

By a suitable choice of the index $n$, it may be assumed that $\alpha^{0}$ is co-exact. Then for all $n, \alpha^{2 n+2}=\delta d \alpha^{2 n}$ and $\alpha^{2 n-2}=\sigma s \alpha^{2 n}$, where $s$ and $\sigma$ are the operators defined in [2]. ${ }^{1}$ The relation between these field systems and equation (1) is given by the following lemma:

Lemma 1. A field system $\left\{\alpha^{n}\right\}$ is contained in two finite-dimensional subspaces of the spaces $C^{p+1}$ and $\Gamma^{p}$ of exact and co-exact forms if and only if there exist constants $c_{0}, \cdots, c_{m}$ with $c_{0}, c_{m} \neq 0$ such that

$$
\sum_{k=0}^{m} c_{k}(\delta d)^{k} \alpha^{0} .=0 .
$$

Proof. If $\left\{\alpha^{2 n}\right\}$ is contained in a finite-dimensional subspace of $\Gamma^{p}$ there exist constants $a_{0}, \cdots, a_{t}$ not all zero such that $\sum_{k=0}^{t} a_{k} \alpha^{2 k}$ $=0$. Letting $a_{r}$ and $a_{r+m}$ be the first and last nonzero constants above, the preceding relation becomes $\sum_{k=0}^{m} a_{r+k}(\delta d)^{k} \alpha^{2 r}=0$; applying the operator $(\sigma s)^{r}$ and writing $c_{k}=a_{r+k}$, one has

$$
\sum_{k=0}^{m} c_{k}(\delta d)^{k} \alpha^{0}=0, \quad \quad \text { with } c_{0}, c_{m} \neq 0 .
$$

Conversely, if the given equation is satisfied, applying the operators $\sigma s$ and $\delta d$ to it one obtains that $\alpha^{-2}$ and $\alpha^{2 m+2}$ are in the linear space spanned by $\alpha^{0}, \cdots, \alpha^{2 m}$, and by induction $\alpha^{2 n}$ is contained in the same subspace.

Theorem 1. If $\alpha^{2 r}=\lambda \alpha^{0}$ for some $\lambda$ and $r$, then $\lambda$ is positive and $\alpha^{2}=\lambda^{1 / r} \alpha^{0}$. 
Proof. Assuming again that $\alpha^{0}$ is co-closed, then $\left(\alpha^{-1}, \alpha^{1}\right)$ $=\left(\alpha^{-1}, d \delta \alpha^{-1}\right)=\left(\delta \alpha^{-1}, \delta \alpha^{-1}\right)=\left(\alpha^{0}, \alpha^{0}\right)$ and similarly $\left(\alpha^{r}, \alpha^{r}\right)=\left(\alpha^{2 r}, \alpha^{0}\right)$ $=\lambda\left(\alpha^{0}, \alpha^{0}\right)$, showing that $\lambda>0$. Similarly, if $\cos \theta_{r, r+2 k}$ is defined by $\left(\alpha^{r}, \alpha^{r+2 k}\right)=\left\|\alpha^{r}\right\| \cdot\left\|\alpha^{r+2 k}\right\| \cos \theta_{r, r+2 k}$, one obtains that

and then

$$
\cos \theta_{-r, r}=\frac{\left\|\alpha^{0}\right\|^{2}}{\left\|\alpha^{-r}\right\| \cdot\left\|\alpha^{r}\right\|}
$$

$$
\cos \theta_{-r, r}=\cos \theta_{-r,-r+2}\left[\frac{\cos ^{2} \theta_{-r+1, r-1}}{\cos \theta_{-r+2, r-2}}\right] \cos \theta_{r-2, r},
$$

since the right hand side of (6) can be expressed by means of (5) in the form

$$
\frac{\left\|\alpha^{-r+1}\right\|^{2}}{\left\|\alpha^{-r}\right\| \cdot\left\|\alpha^{-r+2}\right\|} \cdot \frac{\left\|\alpha^{-r+2}\right\| \cdot\left\|\alpha^{0}\right\|{ }^{2} \cdot\left\|\alpha^{r-2}\right\|}{\left.\left\|\alpha^{-r+1}\right\|\right|^{2} \cdot\left\|\alpha^{r-1}\right\|^{2}} \cdot \frac{\left\|\alpha^{r-1}\right\|^{2}}{\left\|\alpha^{r-2}\right\| \cdot\left\|\alpha^{r}\right\|}
$$

which immediately reduces to the right-hand side of (5). Next, using (6) one can prove by induction that

$$
\cos \theta_{-r, r}=\prod_{i=-r+1}^{r-1}\left(\cos \theta_{i-1, i+1}\right)^{r-|i|} .
$$

Indeed, equation (7) is trivial for $r=1$, and it reduces to (6) when $r=2$. Assuming then that the equation has been proved for $r-2$ and $r-1$, one may substitute the values given by (7) for the quantities in brackets in equation (6). The power of $\cos \theta_{i-1, i+1}$ that results from these substitutions is precisely $2(r-1-|i|)-(r-2-|i|)=r-|i|$ which agrees with (7).

In particular, equation (7) implies that $\cos \theta_{2 r, 0} \leqq \cos \theta_{2,0}$. Since the equation $\alpha^{2 r}=\lambda \alpha^{0}$ is equivalent to $\cos \theta_{2 r, 0}=1$, one must have $\cos \theta_{2,0}$ $=1$ also and hence $\alpha^{2}=\mu \alpha^{0}$ for some $\mu$. Iterating this result one obtains that $\alpha^{2 r}=\mu^{r} \alpha^{0}$ and $\mu^{r}=\lambda$.

The preceding theorem shows that an arbitrary linear relation $\sum_{k=0}^{n} a_{k} \alpha^{2 k}=0$ with $c_{0} c_{n} \neq 0$ need not have a solution. However, if there is a solution, then this solution must also satisfy a linear relation $\sum_{k=0}^{m} c_{k} \alpha^{2 k}=0$ with $c_{0} c_{m} \neq 0$ and $\alpha^{0}, \alpha^{2}, \cdots, \alpha^{2 m-2}$ linearly independent. Such relations will be called minimal and their solutions are described in the following theorems.

THEOREM 2. Let $\alpha$ be a solution of a minimal linear relation $\sum_{k=0}^{m} c_{k}(\delta d)^{k} \alpha=0$ with $c_{0} c_{m} \neq 0$. If the equation $\sum_{k=0}^{m} c_{k} \lambda^{k}=0$ has distinct (nonzero) roots $\lambda_{1}, \cdots, \lambda_{m}$ then there exist forms $\beta_{1}, \cdots, \beta_{m}$ and numbers $b_{1}, \cdots, b_{m}$ such that 


$$
\delta d \beta_{i}=\lambda_{i} \beta_{i}
$$

$$
i=1, \cdots, m \text {, }
$$

and

$$
\alpha=\sum_{i=1}^{m} b_{i} \beta_{i}
$$

Proof. Let $a_{0}, \cdots, a_{m}$ be any numbers that satisfy the conditions $a_{0} \neq 0$ and $a_{m} \neq 0$, and let $\beta=\sum_{i=0}^{m} a_{i} \alpha^{2 i}$, with $\alpha^{2 i}=(\delta d)^{i} \alpha$. Then,

$$
\begin{aligned}
\beta & =\sum_{i=1}^{m}\left(a_{i}-\frac{a_{0}}{c_{0}} c_{i}\right) \alpha^{2 i}, \\
\delta d \beta & =\sum_{i=1}^{m}\left(a_{i-1}-\frac{a_{m}}{c_{m}} c_{i-1}\right) \alpha^{2 i} .
\end{aligned}
$$

Therefore, $\delta d \beta=\lambda \beta$, for some $\lambda$, if and only if

$$
a_{i}-\frac{a_{0}}{c_{0}} c_{i}=\frac{1}{\lambda}\left(a_{i-1}-\frac{a_{m}}{c_{m}} c_{i-1}\right), \quad i=1, \cdots, m .
$$

Since the relevant equations are homogeneous one may use the normalization $a_{0}=c_{0}$ in (10) and then, by induction, one obtains that

$$
a_{s}=c_{s}+\left(1-\frac{a_{m}}{c_{m}}\right) \sum_{i=0}^{s-1} c_{i} \lambda^{i-s}, \quad s=1, \cdots, m .
$$

For $s=m$, this equation reduces to

$$
0=\left(c_{m}-a_{m}\right) \sum_{i=0}^{m} c_{i} \lambda^{i-m} .
$$

If $c_{m}=a_{m}$, then $a_{s}=c_{s}$ for all $s$ and $\beta=0$. If $c_{m} \neq a_{m}$, then (12) implies that (10) has a solution only if $\sum_{i=0}^{m} c_{i} \lambda^{i}=0$, i.e., if $\lambda$ is a solution of the characteristic equation. If this equation is satisfied, $a_{m}$ may be chosen arbitrarily and $a_{s}$ is given by (11) to obtain a solution of (10) and hence a solution of $\delta d \beta=\lambda \beta$. To complete the proof it must be shown that the matrix that gives the $\beta$ 's in terms of the $\alpha$ 's is nonsingular. But from (8) and (11), the $(r, s)$ entry in this matrix is precisely $\left(1-a_{m} / c_{m}\right) \sum_{i=0}^{s-1} c_{i} \lambda^{i-s}$ and this matrix can be obtained by elementary row operations from the matrix with components $c_{0} \lambda_{r}^{-8}$. This matrix is nonsingular if $c_{0} \neq 0$ and if the $\lambda_{r}$ 's are distinct and nonzero.

THEOREM 3. If $\alpha$ satisfies the linear relation $\prod_{i=0}^{m}\left(\delta d-\lambda_{i}\right)^{n_{i}} \alpha=0$, then $\alpha$ also satisfies the linear relation $\prod_{i=0}^{m}\left(\delta d-\lambda_{i}\right) \alpha=0$.

Proof. Let $\beta=\prod_{i=1}^{m}\left(\delta d-\lambda_{i}\right)^{n_{i}} \alpha$, so that $\left(\delta d-\lambda_{0}\right)^{n_{0}} \beta=0$. If $n_{0}=2 n+1$, then 


$$
\begin{aligned}
0 & =\left(\left(\delta d-\lambda_{0}\right)^{2 n+1} \beta,\right. \\
\left.\left(\delta d-\lambda_{0}\right) \beta\right) & =\left\|\left(\delta d-\lambda_{0}\right)^{n+1} \beta\right\|^{2}
\end{aligned}
$$

implying that $\left(\delta d-\lambda_{0}\right)^{n+1} \beta=0$. Similarly, if $n_{0}=2 n$, then

$$
0=\left(\left(\delta d-\lambda_{0}\right)^{2 n} \beta, \beta\right)=\left\|\left(\delta d-\lambda_{0}\right)^{n} \beta\right\|^{2},
$$

implying that $\left(\delta d-\lambda_{0}\right)^{n} \beta=0$. Thus, by iteration one obtains that $\left(\delta d-\lambda_{0}\right) \beta=0$, or $\left(\delta d-\lambda_{0}\right) \prod_{i=1}^{m}\left(\delta d-\lambda_{i}\right)^{n_{i}} \alpha=0$. Since the operators $\left(\delta d-\lambda_{i}\right)$ all commute, the argument can be applied successively to each $\lambda_{i}$ to obtain the desired result.

REMARK. If $\alpha$ is assumed to be co-derived then any root $\lambda_{i}=0$ of the characteristic equation may be neglected since $\delta d \beta=0$ and $\beta=\delta \gamma$ imply $\beta=0$. Thus Theorems 2 and 3 show that the solutions of (1) are linear combinations of the solutions of (3).

The preceding theorems have shown that the solutions of equations (1) and (2) are linear combinations of the solutions of (3) or (4). The remaining results describe some properties of the solutions of these equations. Here it is assumed that $\alpha$ and $\beta$ are both $p$-forms, and the generalized Wronskian $w(\alpha, \beta)$ will be defined by

$$
w(\alpha, \beta)=\frac{\alpha \wedge(d \beta)^{*}}{\|\alpha\| \cdot\|d \beta\|}-\frac{\beta \wedge(d \alpha)^{*}}{\|\beta\| \cdot\|d \alpha\|}+\frac{\delta \alpha \wedge \beta^{*}}{\|\delta \alpha\| \cdot\|\beta\|}-\frac{\delta \beta \wedge \alpha^{*}}{\|\delta \beta\| \cdot\|\alpha\|} .
$$

TheOREM 4. If $d \delta \alpha=a^{2} \alpha, d \delta \beta=b^{2} \beta$ and $a^{2} \neq b^{2}$, then $\alpha \wedge \beta^{*}$ and $\delta \alpha \wedge(\delta \beta)^{*}$ are derived forms. Similarly, if $\delta d \alpha=a^{2} \alpha, \delta d \beta=b^{2} \beta$ and $a^{2} \neq b^{2}$, then $\alpha \wedge \beta^{*}$ and $d \alpha \wedge(d \beta)^{*}$ are derived forms.

Proof. If $d \delta \alpha=a^{2} \alpha$ and $d \delta \beta=b^{2} \beta$, then

$$
\begin{aligned}
& d\left(\delta \alpha \wedge \beta^{*}\right)=a^{2} \alpha \wedge \beta^{*}-\delta \alpha \wedge(\delta \beta)^{*}, \\
& d\left(\delta \beta \wedge \alpha^{*}\right)=b^{2} \beta \wedge \alpha^{*}-\delta \beta \wedge(\delta \alpha)^{*} .
\end{aligned}
$$

Since $\alpha \wedge \beta^{*}=\beta \wedge \alpha^{*}$ and $\delta \alpha \wedge(\delta \beta)^{*}=(\delta \beta) \wedge(\delta \alpha)^{*}$, the preceding equations imply that

$$
\begin{aligned}
\alpha \wedge \beta^{*} & =\left(a^{2}-b^{2}\right)^{-1} d\left[\delta \alpha \wedge \beta^{*}-\delta \beta \wedge \alpha^{*}\right], \\
\delta \alpha \wedge(\delta \beta)^{*} & =\left(a^{2}-b^{2}\right)^{-1} d\left[b^{2} \delta \alpha \wedge \beta^{*}-a^{2} \delta \beta \wedge \alpha^{*}\right] .
\end{aligned}
$$

Similar methods apply when $\delta d \alpha=a^{2} \alpha$ and $\delta d \beta=b^{2} \beta$.

TheOREM 5. If $d \delta \alpha=a^{2} \alpha$ and $d \delta \beta=b^{2} \beta$ with $a, b$ positive, then

$$
\begin{aligned}
& \cos \theta_{\alpha, \beta}=\cos \theta_{\delta \alpha, \delta \beta}, \\
& \cos \theta_{\alpha, \beta}=0
\end{aligned}
$$

if $a^{2} \neq b^{2}$, 


$$
\begin{aligned}
w(\alpha, \beta) & =w(\delta \alpha, \delta \beta), \\
d w(\alpha, \beta) & =(a-b) \frac{\alpha \wedge \beta^{*}}{\|\alpha\| \cdot\|\beta\|}+\frac{\delta \alpha \wedge(\delta \beta)^{*}}{\|\delta \alpha\| \cdot\|\delta \beta\|} .
\end{aligned}
$$

Proof. Since $a^{2}(\alpha, \beta)=(d \delta \alpha, \beta)=(\delta \alpha, \delta \beta)=(\alpha, d \delta \beta)=b^{2}(\alpha, \beta)$, $\cos \theta_{\alpha, \beta}=0$ if $a^{2} \neq b^{2}$, and $\|\delta \alpha\|=a\|\alpha\|,\|\delta \beta\|=b\|\beta\|$. Therefore,

$$
\cos \theta_{\delta \alpha, \delta \beta}=\frac{(\delta \alpha, \delta \beta)}{\|\delta \alpha\| \cdot\|\delta \beta\|}=\cos \theta_{\alpha, \beta} \quad \text { if } a=b .
$$

Also, by definition of $w$,

$$
w(\delta \alpha, \delta \beta)=\frac{\delta \alpha \wedge(d \delta \beta)^{*}}{\|\delta \alpha\| \cdot\|d \delta \beta\|}-\frac{\delta \beta \wedge(d \delta \alpha)^{*}}{\|\delta \beta\| \cdot\|d \delta \alpha\|}=w(\alpha, \beta),
$$

and using equations (13), (14)

$$
d w(\alpha, \beta)=\frac{a^{2} \alpha \wedge \beta^{*}-(\delta \alpha) \wedge(\delta \beta)^{*}}{\|\delta \alpha\| \cdot\|\beta\|}-\frac{b^{2} \alpha \wedge \beta^{*}-(\delta \alpha) \wedge(\delta \beta)^{*}}{\|\delta \beta\| \cdot\|\alpha\|},
$$

which reduces to (iv) using $\|\delta \alpha\|=a \alpha,\|\delta \beta\|=b \beta$.

Appendix. The operators $s, \sigma$.

For each form $\alpha$ of class $C^{\infty}$ defined on the manifolds under consideration, there exists a unique co-derived form $\sigma \alpha$, a unique derived form $s \alpha$ and a unique harmonic form $\alpha_{H}$ such that

$$
\alpha=d \sigma \alpha+\delta s \alpha+\alpha_{H} \text {. }
$$

This equation determines two operators $s$ and $\sigma$ with the following properties:

(1) If $\alpha$ is a $p$-form and $\beta$ is a $p+1$ form, then $(s \alpha, \beta)=(\alpha, \sigma \beta)$.

(2) In the space of derived forms, $s \sigma$ is the inverse of $d \delta$.

(3) In the space of co-derived forms $\sigma s$ is the inverse of $\delta a$

\section{BIBLIOGRAPHY}

1. G. de Rham and K. Kodaira, Harmonic integrals, Institute for Advanced Studies seminar notes (mimeographed), Princeton, 1950.

2. R. A. Restrepo, Formal anti-derivatives on manifolds, to be published.

University of British COlumbia 